\title{
The Difference in Maximum Standardized Uptake Value among Lung Adenocarcinomas Located at the Upper and Lower Zone on PET/CT
}

\author{
Tomoharu Yoshiya ${ }^{a}$ Yoshihiro Miyata ${ }^{a}$ Yuta Ibuki ${ }^{a}$ Takahiro Mimae ${ }^{a}$ \\ Yasuhiro Tsutani $^{a}$ Haruhiko Nakayama $^{b}$ Sakae Okumurac \\ Masahiro Yoshimurad Morihito Okada ${ }^{\mathrm{a}}$ \\ ${ }^{a}$ Department of Surgical Oncology, Hiroshima University, Hiroshima, ${ }^{b}$ Department of Thoracic Surgery, Kanagawa \\ Cancer Center, Yokohama, ${ }^{C}$ Department of Thoracic Surgery, Cancer Institute Hospital, Tokyo, and ${ }^{\mathrm{d}}$ Department of \\ Thoracic Surgery, Hyogo Cancer Center, Akashi, Japan
}

\section{Key Words \\ Lung carcinoma - Positron emission tomography imaging in lung cancer $\cdot$ Location}

\begin{abstract}
Background: Findings on ${ }^{18} \mathrm{~F}$-fluorodeoxyglucose positron emission tomography/computed tomography (PET/CT) are surrogate markers of malignancy in lung adenocarcinoma. Breathing during PET/CT can substantially reduce the maximum standardized uptake value (maxSUV) of lung tumors when they are located at the lower zone (LZ). Objectives: We assessed whether lung cancer location influences the malignancy predicted by maxSUV. Methods: 608 patients with clinical stage IA lung adenocarcinoma had been preoperatively examined by PET/CT and high-resolution computed tomography (HRCT). We evaluated the clinicopathological characteristics of these patients and the accuracy of precognition obtained by maxSUV between the upper zone (UZ, $n=395)$ and the $L Z(n=213)$. maxSUV was also analyzed for matched pairs between the two groups. Results: The mean maxSUV in the LZ group was significantly lower than that in the UZ group ( $1.98 \pm 1.73$ vs. $2.44 \pm 2.43$, respectively; $p=$ $0.0145)$. The receiver operating characteristics curve of
\end{abstract}

maxSUV for predicting high-grade malignancy (lymphatic, vascular, pleural invasion, or lymph node metastasis) was larger for the UZ group than for the LZ group [0.89, 95\% confidence interval $(\mathrm{Cl}) 0.86-0.93$, vs. $0.82,95 \% \mathrm{Cl} 0.76-0.88$ ]. Analysis for maxSUV of 213 pairs matched for the solid component size on HRCT, pathological characteristics, and gender revealed that maxSUV in the LZ group was significantly lower than that in the UZ group $(1.98 \pm 1.73$ vs. $2.47 \pm 2.39$, respectively; $p<0.001)$. Conclusions: maxSUV of a tumor in the LZ group is apparently lower than the value which reflects the potential malignancy of a tumor. We have to carefully consider these facts when selecting the appropriate surgical procedure for lung cancer with PET/CT and HRCT.

(c) 2015 S. Karger AG, Basel

\section{Introduction}

It has been well recognized that ${ }^{18} \mathrm{~F}$-fluorodeoxyglucose positron emission tomography/computed tomography (PET/CT) is valuable for the diagnosis and staging of lung cancer. The maximum standardized uptake value (maxSUV) reflects pathological malignancy $[1,2]$. It helps to determine the optimum treatment, including the

\section{KARGER 125}

C 2015 S. Karger AG, Base

$0025-7931 / 15 / 0904-0293 \$ 39.50 / 0$

E-Mail karger@karger.com

www.karger.com/res
Morihito Okada MD, PhD

Department of Surgical Oncology

Research Institute for Radiation Biology and Medicine, Hiroshima University

1-2-3 Kasumi, Minami-ku, Hiroshima City, Hiroshima 734-0037 (Japan)

E-Mail morihito@hiroshima-u.ac.jp 
extent of surgical intervention, chemotherapy, and radiation. Several reports have described that the respiratory motion of the diaphragm affected computed tomographic images and maxSUV on PET/CT in the chest and abdomen. It greatly affects maxSUV in the lower lung field [3-5].

High-resolution CT (HRCT) has recently advanced, and CT screening is widely spread. Therefore, the discovery of small lung cancers, especially adenocarcinomas, has been increasing. In early-stage lung adenocarcinomas, it is of great significance to accurately predict the potential malignancy of the tumors in the preoperative setting when making a decision on the appropriate procedure, including lobectomy and sublobar resections. If the difference in maxSUV in tumors between the upper (UZ) and lower zone (LZ) is confirmed, even when the malignancy grade is matched, we should take this difference into consideration and need to cautiously predict the potential malignancy of the tumors.

The purpose of this retrospective study is to compare the predictability of the pathological findings on the basis of maxSUV and determine the difference in maxSUV, when the patients' characteristics and malignancy of the tumor are matched, between the UZ and LZ in patients with clinical stage IA lung adenocarcinomas.

\section{Materials and Methods}

We enrolled 608 patients with clinical IA lung adenocarcinomas, of whom the located segment of the tumors was known, at 4 institutions (Hiroshima University, Kanagawa Cancer Center, Cancer Institute Hospital, and Hyogo Cancer Center) from August 1, 2005 to June 30, 2010. The database has been maintained prospectively, and patients' data were obtained retrospectively from multicenter databases. Patients with incompletely resected tumors (R1 or R2) and those with multiple tumors or a history of previous lung cancer operations were excluded from this database. Before complete R0 resection, PET/CT and HRCT were taken for all patients. We did not ask the patients to hold a deep inspiration breath during the tomography. maxSUV was evaluated independently by each institutional radiologist.

We defined the lung segments 1, 2, 3, and 6 as UZ and segments $4,5,7,8,9$, and 10 as LZ. The stages of the tumors were classified according to the 7th edition of the TNM [6]. In case HRCT revealed a swelling lymph node and PET/CT showed no accumulation at the mediastinal or hilar lymph node, endobronchial ultrasonography or mediastinoscopy for detecting lymph node metastases were not performed. Patients with pathological lymph node metastases received postoperative platinum-based chemotherapy. Surgically resected tumors were fixed in $10 \%$ formalin and embedded in paraffin. Sections of the largest cut of tumors and lymph node with hematoxylin-eosin and elastica van Gieson staining were evaluated histopathologically, including lymphatic invasion
(Ly), vascular invasion (V), pleural invasion (Pl), and lymph node metastases (N). Each Institutional Review Board granted approval for this study. Informed consent from the individual patients was not required because this was a retrospective review of medical records from multicenter databases prospectively maintained.

\section{High-Resolution Computed Tomography}

Chest images were obtained using 16-row multidetector CT, which was independent of subsequent ${ }^{18} \mathrm{~F}$-fluorodeoxyglucose $\mathrm{PET} / \mathrm{CT}$ examinations. High-resolution images of the tumors were acquired using the following parameters: $120 \mathrm{kVp}, 200 \mathrm{~mA}$, section thickness $1-2 \mathrm{~mm}$, pixel resolution $512 \times 512$, scanning time $0.5-1 \mathrm{~s}$, a high spatial reconstruction algorithm with a $20-\mathrm{cm}$ field of view (FOV), and mediastinal (level, $40 \mathrm{HU}$; width, $400 \mathrm{HU}$ ) and lung (level, $-600 \mathrm{HU}$; width, 1,600 HU) window settings.

\section{${ }^{18}$ F-Fluorodeoxyglucose Positron Emission Tomography/ Computed Tomography}

The patients were instructed to fast for more than $4 \mathrm{~h}$ prior to an intravenous injection of $74-370 \mathrm{MBq}$ of ${ }^{18} \mathrm{~F}$-fluorodeoxyglucose. Their blood glucose level was confirmed to be $<150 \mathrm{mg} / \mathrm{dl}$ before injection. Patients with blood glucose values $\geq 150 \mathrm{mg} / \mathrm{dl}$ were excluded. Sixty minutes after the intravenous injection of ${ }^{18} \mathrm{~F}$ fluorodeoxyglucose, all patients were positioned in a supine position on the imaging table and were instructed to breathe freely. The images were obtained using Discovery ST (GE Healthcare, Little Chalfont, UK), Aquiduo (Toshiba Medical Systems Corporation, Tochigi, Japan), or Biograph Sensation 16 (Siemens Healthcare, Erlangen, Germany) integrated PET/CT scanners. Hiroshima University was the control institute. Low-dose unenhanced CT images of 2- to 4-mm section thickness for attenuation correction and localization of lesions were obtained from the head to the pelvic floor of each patient using a standard protocol. Immediately after CT, PET covered the identical axial FOV for 2-4 min per table position depending on the condition of the patient and scanner performance. Both CT and PET studies proceeded during normal tidal breathing. All PET images with a $50-\mathrm{cm}$ FOV were reconstructed using a FORE-OSEM algorithm with CT-derived attenuation correction.

We used an International Electrotechnical Commission body phantom set corresponding to the NU 2-2001 standard published by the National Electrical Manufacturers Association (NEMA). Variations in SUV among institutions were minimized using an anthropomorphic body phantom and 6 spheres (inner diameter, $10,13,17,22,28$, and $37 \mathrm{~mm}$ ). From the phantom study, a calibration factor was calculated by dividing the actual SUV by the measured mean SUV in the phantom background to reduce interinstitutional SUV variability. The final SUV is referred to as the revised maxSUV [7, 8]. Adjustment of interinstitutional variability in SUV narrowed the range from $0.89-1.24$ to $0.97-1.18$ when the maxSUV ratio was expressed as the maxSUV reported by each institute relative to the maxSUV reported by the control institute.

\section{Statistical Analysis}

Clinicopathological characteristics were reported to the patients descriptively. Data are presented as numbers (mean \pm standard deviation) or percentages unless otherwise stated. The t test or Mann-Whitney U test were used to compare continuous variables in all cohort patients. Frequencies were compared using the $\chi^{2}$ test or Fisher's exact test for categorical variables in all cohort 
Table 1. Clinicopathological characteristics

\begin{tabular}{lccl}
\hline & $\begin{array}{l}\mathrm{UZ} \\
(\mathrm{n}=395)\end{array}$ & $\begin{array}{l}\mathrm{LZ} \\
(\mathrm{n}=213)\end{array}$ & p value \\
\hline Age, years & $65.7 \pm 9.7$ & $65.8 \pm 9.6$ & 0.867 \\
Sex & & & \\
$\quad$ Male & $188(48)$ & $80(38)$ & 0.021 \\
$\quad$ Female & $207(52)$ & $133(62)$ & \\
Whole tumor size, mm & $19.7 \pm 5.8$ & $19.0 \pm 5.9$ & 0.241 \\
SCS ${ }^{1}$ mm & $11.5 \pm 8.8$ & $12.1 \pm 8.4$ & 0.44 \\
maxSUV & $2.44 \pm 2.43$ & $1.98 \pm 1.73$ & 0.0145 \\
Ly & $54(14)$ & $35(16)$ & 0.40 \\
V & $71(18)$ & $32(15)$ & 0.37 \\
Pl & $45(11)$ & $20(9.4)$ & 0.493 \\
N & $25(6.3)$ & $16(7.5)$ & 0.613 \\
BAC ratio ${ }^{2}, \%$ & $49.8 \pm 36.8$ & $45.3 \pm 36.7$ & 0.155 \\
\hline
\end{tabular}

Figures are means \pm SD or numbers with percentages in parentheses.

${ }^{1}$ Maximum diameter of the solid component on HRCT.

${ }^{2}$ Ratio of BAC in the whole tumor mass.

patients when appropriate. Pathological high-grade malignancy was defined as including more than one positive pathological factor in Ly, $\mathrm{V}, \mathrm{Pl}$, or $\mathrm{N}$. Receiver operating characteristics (ROC) curves of maxSUV were used for predicting Ly, V, Pl, N, and highgrade malignancy. For analyses of matched-pair patients, the Mantel-Haenszel $\chi^{2}$ test with continuity correction was used. $\mathrm{p}<0.05$ was considered statistically significant. All statistical analyses were performed with EZR (Saitama Medical Centre, Jichi Medical University, Tokyo, 2012), which is a graphical user interface for $\mathrm{R}$ (The $\mathrm{R}$ Foundation for Statistical Computing, version 2.13.0).

\section{Results}

There were no significant differences between $\mathrm{UZ}$ and LZ patients in the whole tumor size, the solid component size (SCS) on HRCT, which is defined as the maximum dimension of the solid component excluding the groundglass opacity, Ly, $\mathrm{V}, \mathrm{Pl}, \mathrm{N}$, and bronchioloalveolar carcinoma (BAC) ratio, which represents the ratio that the BAC occupies in the whole tumor mass. In the UZ group, there were more male patients than in the LZ group ( $p=0.021)$. The mean maxSUV of tumors in the LZ group was significantly lower than that of tumors in the UZ group (1.98 \pm 1.73 vs. $2.44 \pm 2.43$, respectively; $\mathrm{p}=0.0145$; table 1$)$.

ROC analysis for maxSUV to predict Ly, V, Pl, N, and high-grade malignancy revealed that the area under the curve was larger in the UZ group than in the LZ group (table 2; fig. 1). Cutoff values of maxSUV, sensitivity, and specificity for predicting each pathological factor in the
Table 2. ROC analysis for maxSUV to predict pathological findings

\begin{tabular}{lll}
\hline Variable & UZ & LZ \\
& & \\
& & \\
\hline Ly & $0.84(0.80-0.89)$ & $0.78(0.69-0.86)$ \\
& $2.40(0.87 / 0.75)$ & $1.87(0.80 / 0.66)$ \\
\hline $\mathrm{V}$ & $0.88(0.85-0.93)$ & $0.84(0.78-0.90)$ \\
& $2.53(0.87 / 0.81)$ & $1.88(0.88 / 0.68)$ \\
\hline $\mathrm{Pl}$ & $0.87(0.83-0.91)$ & $0.72(0.59-0.85)$ \\
& $2.33(0.93 / 0.73)$ & $1.87(0.78 / 0.63)$ \\
\hline $\mathrm{N}$ & $0.81(0.75-0.87)$ & $0.79(0.71-0.87)$ \\
& $2.71(0.84 / 0.74)$ & $1.59(1.00 / 0.55)$ \\
\hline High-grade malignancy ${ }^{1}$ & $0.89(0.86-0.93)$ & $0.82(0.76-0.88)$ \\
& $2.30(0.85 / 0.83)$ & $1.87(0.79 / 0.75)$ \\
\hline
\end{tabular}

Figures are either area under the curve with 95\% confidence interval in parentheses or cutoff value with sensitivity/specificity in parentheses.

${ }^{1}$ Positive for Ly, V, Pl, or $\mathrm{N}$.

Table 3. Comparison of maxSUV between the two groups after matching for gender, SCS, Ly, V, Pl, and N

\begin{tabular}{lccl}
\hline & $\begin{array}{l}\text { UZ } \\
(\mathrm{n}=213)\end{array}$ & $\begin{array}{l}\text { LZ } \\
(\mathrm{n}=213)\end{array}$ & p value \\
\hline Age, years & $65.7 \pm 9.4$ & $66 \pm 9.6$ & 0.84 \\
Sex, male/female & $83 / 130$ & $80 / 133$ & 0.248 \\
SCS, mm & $12.2 \pm 8.4$ & $12.1 \pm 8.4$ & 0.18 \\
Ly & $33(15)$ & $35(16)$ & 0.48 \\
V & $32(15)$ & $32(15)$ & 1 \\
Pl & $20(9)$ & $20(9)$ & 1 \\
N & $16(7.5)$ & $16(7.5)$ & 1 \\
maxSUV & $2.47 \pm 2.39$ & $1.98 \pm 1.73$ & $<0.001$ \\
\hline
\end{tabular}

Figures are means \pm SD or numbers with percentages in parentheses unless otherwise indicated.

UZ group were higher than those in the LZ group (table 2). Therefore, the predictability of all outcomes on the basis of maxSUV seemed to be better in the UZ group than in the LZ group.

Analysis for maxSUV of 213 pairs matched for the SCS on HRCT, each pathological factor ( $\mathrm{Ly}, \mathrm{V}, \mathrm{Pl}$, and $\mathrm{N}$ ), and gender confirmed that maxSUV of tumors in the LZ group was significantly lower than that of tumors in the UZ group (2.47 vs. 1.98, respectively; $\mathrm{p}<0.001$; table 3 ).

On the other hand, analysis for high-grade malignancy of 213 pairs matched for maxSUV, the SCS on HRCT, and gender confirmed that tumors in the LZ group were sig- 
Fig. 1. ROC area under the curve to predict high-grade malignancy. a ROC area under the curve to predict high-grade malignancy (positive for Ly, V, PI, or N) in the UZ. b ROC area under the curve to predict high-grade malignancy in the LZ.

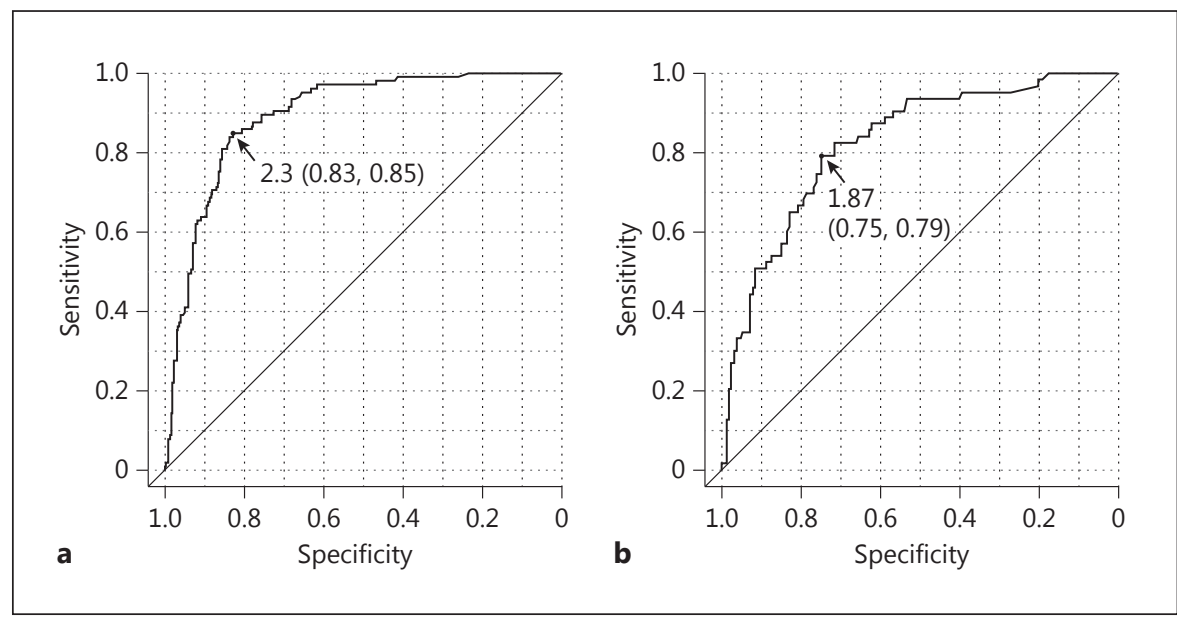

Table 4. Comparison of high-grade malignancy between the two groups after matching for gender, maxSUV, and SCS

\begin{tabular}{lrrl}
\hline & $\begin{array}{l}\mathrm{UZ} \\
(\mathrm{n}=213)\end{array}$ & $\begin{array}{l}\mathrm{LZ} \\
(\mathrm{n}=213)\end{array}$ & p value \\
\hline Age, years & $66.0 \pm 9.9$ & $66.3 \pm 9.9$ & 0.94 \\
Sex, male/female & $83 / 130$ & $80 / 133$ & 0.248 \\
SCS, mm & $11.7 \pm 8.4$ & $12.1 \pm 8.4$ & 0.8025 \\
maxSUV & $1.97 \pm 1.6$ & $1.98 \pm 1.7$ & 0.80 \\
High-grade malignancy $^{1}$ & $47(22)$ & $63(30)$ & 0.0412 \\
\hline
\end{tabular}

Figures are means \pm SD or numbers with percentages in parentheses unless otherwise indicated.

${ }^{1}$ Positive for Ly, V, Pl, or $\mathrm{N}$.

nificantly malignant compared to those in the UZ group $(\mathrm{p}=0.0412)$. The frequency for high-grade malignancy was $22 \%(n=47 / 213)$ in the UZ group and 30\% ( $n=$ $63 / 213$ ) in the LZ group (table 4).

\section{Discussion}

Our study on 608 patients with clinical stage IA lung adenocarcinomas admitted in 4 institutions revealed that the location of the lung tumor affected the reading of maxSUV on PET/CT. The predictability of pathological findings on the basis of maxSUV was better in the UZ group than in the LZ group. maxSUV obtained from the latter group were lower than those from the former group, even when the patients' characteristics and the tumor malignancy were matched. Thus, we concluded that maxSUV of tumors in the LZ group is apparently lower than that which reflects the potential malignancy of tumors.

The PET component of PET/CT takes several minutes so that it comprises images averaged out of many breathing cycles. It is well known that PET/CT images, as well as HRCT, are generally affected by the respiratory motion. Lesions were sometimes mislocated during normal respiratory movement $[5,9,10]$. In addition, respiratory motion results in the underestimation of a measured SUV of lesions, blurring of tumor location, overestimation of tumor volume, and mismatching of PET and CT images $[9,11-13]$. To resolve these problems, several methods have been developed, including respiratory-gated PET/ CT [11], deep inspiration breath hold PET/CT [14-16], and motion-corrected PET reconstruction [17]. These studies indicated an underestimation of the mean and/or maxSUV of tumors, especially in the lower lung field. They demonstrated that minimizing artifacts due to the diaphragm movement improved the tumor quantitation and localization on the PET/CT images and increased maxSUV. Liu et al. [18] used a respiratory gating system of real-time position management on PET/CT. They evaluated the impact of respiratory motion in a routine clinical practice and signified the importance of capturing variations in respiratory motion patterns.

The accurate measurement of maxSUV for better treatment is important in the preoperative evaluation for potential malignancy of the lesions $[19,20]$. When maxSUV obtained with the above-mentioned methods was compared with that of free breathing, the difference in maxSUV of a tumor located in the lower lung field was higher than that of a tumor in the upper lung field. Furthermore, the difference in maxSUV of a small tumor was 
higher than that of a large tumor. However, the reports did not define the segment where the respiratory motion showed most effect. We defined segments $1,2,3$, and 6 as UZ and segments $4,5,7,8,9$, and 10 as $\mathrm{LZ}$ on the premise that segment 6 is located behind the upper lobe on the axial view of the HRCT. The comparison of maxSUV by this definition revealed a significant difference.

We have previously reported the benefits of using SCS on HRCT compared with considering the whole tumor size for predicting the malignancy and prognosis of clinical stage IA lung adenocarcinoma [1]. In addition, we have proved that maxSUV on PET/CT and SCS on HRCT are considerable factors for predicting pathological malignancy and prognosis in patients with clinical stage IA lung adenocarcinoma $[1,2,8]$. However, if there is a significant difference in maxSUV between the two groups due to the location of the tumors, it is a great concern for physicians. We performed two matching analyses to examine this problem.

The purpose of the first analysis was to examine whether there was a significant difference in maxSUV between the two groups when the factors reflecting the malignancy grade of tumors were matched. The UZ group had more male patients so that we matched gender, SCS on HRCT, and each pathological finding (Ly, V, PI, and N). The analysis confirmed that maxSUV of tumors in the LZ was significantly lower than that in the UZ. The purpose of the second analysis was to examine whether there was a significant difference in the pathological malignancy between the two groups when the preoperative factors reflecting the malignancy grade of tumors were matched. Gender, maxSUV, and SCS on HRCT were matched. Based on our previous reports, we needed to match not only maxSUV but also SCS on HRCT in order to reconcile the expected pathological malignancy. This analysis confirmed that tumors in the LZ were significantly malignant compared to those in the UZ.

One limitation of this study is the wide variation in maxSUV among multiple institutions. Many factors such as preparation procedures, scan acquisition, image reconstruction, and data analysis can affect maxSUV. In the present study, an anthropomorphic body phantom was used to minimize the interinstitutional variability in maxSUV. Another limitation is that PET/CT in the present study was performed without respiratory motion correction. Although we are aware of the importance of a respiratory gating system of real-time position management [18] and 4D-PET/CT by low-dose interpolated CT for attenuation correction [21], it is difficult for thoracic surgeons to perform these methods every time in a clinical setting. Therefore, we examined the difference in

Location of Lung Cancer and PET/CT Image
maxSUV between the UZ and the LZ with only 3D-PET/ CT on patients with clinical stage IA lung adenocarcinomas.

The standard surgical procedure for resectable nonsmall cell lung cancer (NSCLC) is a lobectomy with lymph node dissection [22]. A study from the Surveillance Epidemiology and End Results database revealed that lobectomy correlated with a significant advantage in comparison with segmentectomy in patients with stage I NSCLC [23]. On the other hand, recent advances in HRCT and PET/CT can help detect small lung cancers, especially adenocarcinomas. Several studies have reported that the survival rate was not different between patients treated with segmentectomy or lobectomy for small peripheral NSCLC [24-28]. Segmentectomy is a less invasive surgery than lobectomy with respect to preservation of the pulmonary function [29], while the incidence of locoregional recurrence is a little higher after segmentectomy than that after lobectomy [30]. Therefore, it is of great significance to accurately predict the potential malignancy of tumors in the preoperative setting to determine an appropriate surgical procedure. Although SCS on HRCT and maxSUV on PET/CT are useful for predicting the pathological malignancy and prognosis in early-stage lung adenocarcinoma $[1,2,8,10]$, the findings of the present study with the usual 3D-PET/CT had not been taken into account in the previous studies. In this study, the predictability for each pathological factor and high-grade malignancy on the basis of maxSUV on ROC analyses seems to be better in the UZ group than in the LZ group. Cutoff values of maxSUV for predicting high-grade malignancy are 2.30 in the UZ group and 1.87 in the LZ group. The suitable surgical procedure may be lobectomy for tumors in the UZ with maxSUV $>2.30$ and for tumors in the LZ with maxSUV >1.87 in patients with clinical stage IA lung adenocarcinomas.

In conclusion, maxSUV of tumors in the LZ group is lower than the value that reflects the potential malignancy of tumors. We should take the underestimation of maxSUV in the LZ group into consideration in the preoperative setting.

\section{Acknowledgement}

We thank Kozaburo Hayashi for reviewing the manuscript.

\section{Financial Disclosure and Conflicts of Interest}

The authors have declared no conflicts of interest or any funding for this study. 


\section{References}

-1 Tsutani Y, Miyata Y, Nakayama H, Okumura S, Adachi S, Yoshimura M, et al: Prognostic significance of using solid versus whole tumor size on high-resolution computed tomography for predicting pathologic malignant grade of tumors in clinical stage IA lung adenocarcinoma: a multicenter study. J Thorac Cardiovasc Surg 2012;143:607-612.

-2 Tsutani Y, Miyata Y, Yamanaka T, Nakayama H, Okumura S, Adachi S, et al: Solid tumors versus mixed tumors with a ground-glass opacity component in patients with clinical stage IA lung adenocarcinoma: prognostic comparison using high-resolution computed tomography findings. J Thorac Cardiovasc Surg 2013;146:17-23.

-3 Nakamoto Y, Tatsumi M, Cohade C, Osman M, Marshall LT, Wahl RL: Accuracy of image fusion of normal upper abdominal organs visualized with PET/CT. Eur J Nucl Med Mol Imaging 2003;30:597-602.

$\checkmark 4$ Osman MM, Cohade C, Nakamoto Y, Marshall LT, Leal JP, Wahl RL: Clinically significant inaccurate localization of lesions with PET/CT: frequency in 300 patients. J Nucl Med 2003;44:240-243.

$\checkmark 5$ Pan T, Mawlawi O, Nehmeh SA, Erdi YE, Luo $\mathrm{D}$, Liu $\mathrm{HH}$, et al: Attenuation correction of PET images with respiration-averaged CT images in PET/CT. J Nucl Med 2005;46:14811487.

$\checkmark 6$ Goldstraw P, Crowley J, Chansky K, Giroux DJ, Groome PA, Rami-Porta R: The IASLC lung cancer staging project: proposals for the revision of the TNM stage groupings in the forthcoming (seventh) edition of the TNM classification of malignant tumours. J Thorac Oncol 2007;2:706-714.

7 Nakayama H, Okumura S, Daisaki H, Kato Y, Uehara H, Adachi S: Value of integrated positron emission tomography revised using a phantom study to evaluate malignancy grade of lung adenocarcinoma: a multicenter study. Cancer 2010;116:3170-3177.

>8 Okada M, Nakayama H, Okumura S, Daisaki $\mathrm{H}$, Adachi S, Yoshimura M: Multicenter analysis of high-resolution computed tomography and positron emission tomography/computed tomography findings to choose therapeutic strategies for clinical stage IA lung adenocarcinoma. J Thorac Cardiovasc Surg 2011;141:1384-1391.
$>9$ Nehmeh SA, Erdi YE, Ling CC, Rosenzweig $\mathrm{KE}$, Squire OD, Braban LE, et al: Effect of respiratory gating on reducing lung motion artifacts in PET imaging of lung cancer. Med Phys 2002;29:366-371.

$\checkmark 10$ Erdi YE, Nehmeh SA, Pan T, Pevsner A, Rosenzweig KE, Mageras G, et al: The CT motion quantitation of lung lesions and its impact on PET-measured SUVs. J Nucl Med 2004;45:1287-1292.

11 Nehmeh SA, Erdi YE, Pan T, Pevsner A, Rosenzweig KE, Yorke E, et al: Four-dimensional (4D) PET/CT imaging of the thorax. Med Phys 2004;31:3179-3186.

$>12$ Nehmeh SA, Erdi YE, Ling CC, Rosenzweig KE, Schoder H, Larson SM, et al: Effect of respiratory gating on quantifying PET images of lung cancer. J Nucl Med 2002;43:876-881.

$>13$ Nehmeh SA, Erdi YE: Respiratory motion in positron emission tomography/computed tomography: a review. Semin Nucl Med 2008; 38:167-176.

14 Nehmeh SA, Erdi YE, Meirelles GS, Squire O, Larson SM, Humm JL, et al: Deep-inspiration breath-hold PET/CT of the thorax. J Nucl Med 2007;48:22-26.

15 Kawano T, Ohtake E, Inoue T: Deep-inspiration breath-hold PET/CT of lung cancer: maximum standardized uptake value analysis of 108 patients. J Nucl Med 2008;49:12231231 .

16 Torizuka T, Tanizaki Y, Kanno T, Futatsubashi M, Yoshikawa E, Okada H, et al: Single 20-second acquisition of deep-inspiration breath-hold PET/CT: clinical feasibility for lung cancer. J Nucl Med 2009;50:1579-1584.

-17 Lamare F, Ledesma Carbayo MJ, Cresson T, Kontaxakis G, Santos A, Le Rest CC, et al: List-mode-based reconstruction for respiratory motion correction in PET using non-rigid body transformations. Phys Med Biol 2007; 52:5187-5204.

18 Liu C, Pierce LA 2nd, Alessio AM, Kinahan PE: The impact of respiratory motion on tumor quantification and delineation in static PET/CT imaging. Phys Med Biol 2009;54: 7345-7362.

19 Dewan NA, Gupta NC, Redepenning LS, Phalen JJ, Frick MP: Diagnostic efficacy of PETFDG imaging in solitary pulmonary nodules. Potential role in evaluation and management. Chest 1993;104:997-1002.
20 Brocken P, van der Heijden HF, Dekhuijzen PN, Peters-Bax L, de Geus-Oei LF: High performance of ${ }^{18} \mathrm{~F}$-fluorodeoxyglucose positron emission tomography and contrast-enhanced CT in a rapid outpatient diagnostic program for patients with suspected lung cancer. Respiration 2014;87:32-37.

21 Huang TC, Wang YC, Kao CH: Thoracic tumor volume delineation in 4D-PET/CT by low dose interpolated CT for attenuation correction. PLoS One 2013;8:e75903.

22 Watanabe S: Lymph node dissection for lung cancer: past, present, and future. Gen Thorac Cardiovasc Surg 2014;62:407-414.

23 Whitson BA, Groth SS, Andrade RS, Maddaus MA, Habermann EB, D’Cunha J: Survival after lobectomy versus segmentectomy for stage I non-small cell lung cancer: a population-based analysis. Ann Thorac Surg 2011;92:1943-1950.

24 Jensik RJ, Faber LP, Milloy FJ, Monson DO: Segmental resection for lung cancer. A fifteen-year experience. J Thorac Cardiovasc Surg 1973;66:563-572.

25 Kodama K, Doi O, Higashiyama M, Yokouchi $\mathrm{H}$ : Intentional limited resection for selected patients with T1 N0 M0 non-small-cell lung cancer: a single-institution study. J Thorac Cardiovasc Surg 1997;114:347-353.

26 Okada M, Yoshikawa K, Hatta T, Tsubota N Is segmentectomy with lymph node assessment an alternative to lobectomy for nonsmall cell lung cancer of $2 \mathrm{~cm}$ or smaller? Ann Thorac Surg 2001;71:956-960.

-27 Yoshikawa K, Tsubota N, Kodama K, Ayabe H, Taki T, Mori T: Prospective study of extended segmentectomy for small lung tumors: the final report. Ann Thorac Surg 2002;73: 1055-1058, discussion 1058-1059.

28 Okada M, Koike T, Higashiyama M, Yamato Y, Kodama K, Tsubota N, et al: Radical sublobar resection for small-sized non-small cell lung cancer: a multicenter study. J Thorac Cardiovasc Surg 2006;132:769-775.

29 Ohtaki Y, Shimizu K: Anatomical thoracoscopic segmentectomy for lung cancer. Gen Thorac Cardiovasc Surg 2014;62:586-593.

30 Sawabata N: Locoregional recurrence after pulmonary sublobar resection of non-small cell lung cancer: can it be reduced by considering cancer cells at the surgical margin? Gen Thorac Cardiovasc Surg 2013;61:9-16. 